\title{
The American Joint Committee on Cancer: the 7th Edition of the AJCC Cancer Staging Manual and the Future of TNM
}

\author{
Stephen B. Edge, $\mathrm{MD}^{1,2}$ and Carolyn C. Compton, $\mathrm{MD}, \mathrm{PhD}^{1,3}$ \\ ${ }^{1}$ American Joint Committee on Cancer, Chicago, IL; ${ }^{2}$ Department of Surgical Oncology, Roswell Park Cancer Institute, \\ Buffalo, NY; ${ }^{3}$ National Cancer Institute, Bethesda, MD
}

\begin{abstract}
The American Joint Committee on Cancer and the International Union for Cancer Control update the tumor-node-metastasis (TNM) cancer staging system periodically. The most recent revision is the 7th edition, effective for cancers diagnosed on or after January 1, 2010. This editorial summarizes the background of the current revision and outlines the major issues revised. Most notable are the marked increase in the use of international datasets for more highly evidenced-based changes in staging, and the enhanced use of nonanatomic prognostic factors in defining the stage grouping. The future of cancer staging lies in the use of enhanced registry data standards to support personalization of cancer care through cancer outcome prediction models and nomograms.
\end{abstract}

The extent of a cancer at time of diagnosis is a key factor used to define treatment and to assess the chance of successful treatment outcome. Cancer staging systems codify the extent of cancer to provide clinicians and patients with the means to quantify prognosis for individual patients and to compare groups of patients in clinical trials and who receive standard care around the world. There are three staging systems used in the USA. Some state cancer registries used for population incidence and surveillance employ the relatively simply Summary Stage System. The National Cancer Institute Surveillance, Epidemiology, and End Results (SEER) program developed its own "extent of disease" system. The most widely used staging system among clinicians is the TNM system maintained by the American Joint Committee on Cancer (AJCC) and the International Union for Cancer Control (UICC). This

(C) Society of Surgical Oncology 2010

Published Online: 24 February 2010

S. B. Edge, MD

e-mail: stephen.edge@ roswellpark.org system codes the extent of the primary tumor $(\mathrm{T})$, regional lymph nodes $(\mathrm{N})$, and distant metastases $(\mathrm{M})$ and provides a "stage grouping" based on $\mathrm{T}, \mathrm{N}$, and $\mathrm{M}$.

TNM is updated periodically based on advances in understanding of advances of cancer prognosis to remain current and relevant to clinical practice. The latest revision of TNM, presented in the 7th edition of the AJCC Cancer Staging Manual, takes effect for cases diagnosed on or after January 1, 2010. ${ }^{1}$ This supplants the 6th edition, in use since 2003. Beginning in this volume, the Annals of Surgical Oncology is publishing a series of editorials that highlight the major changes in staging presented in the 7 th edition of TNM.

Publication of the 7th edition of the AJCC Cancer Staging Manual comes at a major watershed for cancer staging and the AJCC. First, the revolution in understanding cancer biology is dramatically altering clinical oncology. Historically, cancer staging and treatment planning was based solely on the anatomic extent of the cancer. Anatomy continues to be a key prognostic factor for cancer, and anatomic-based staging will remain critically important. However the rapidly increasing specific knowledge of cancer biology provides prognostic information that complements and in some cases is more relevant than anatomic extent. This information must be incorporated into cancer prognostic models, and into the TNM nomenclature, if staging systems are to remain useful for patients and clinicians. Maintaining clear systems for defining anatomic stage while including biologic prognostic information is a key challenge and opportunity for the AJCC.

Secondly, the rise in the availability and use of electronic records is revolutionizing the capacity to capture, store, and use detailed information on cancer. This will lead to structured data tools integrated with clinical care systems that allow real-time data capture and provide immediate support to clinicians. In addition, the 
availability of highly granular data including biologic information will hasten the development of new prognostic and predictive models that provide individual precise outcome prediction that supplants stage groupings in clinical use. Such models are already in use for some common diseases. A prime example is Adjuvantonline ${ }^{\circledR}$, a prognostic and predictive model to assist clinicians and patients in decisions regarding adjuvant therapy in breast, colon, and lung cancer. ${ }^{2}$ Developing and supporting implementation of electronic data capture and analytic tools and their incorporation into patient care documentation is another important opportunity for the AJCC.

With the publication of the 7th edition of the AJCC Cancer Staging Manual and related products, the AJCC is taking important steps to use these opportunities to improve patient care. The 7th edition extends the use of nonanatomic factors in cancer staging where supported by clear evidence. In addition, the 7th edition of the AJCC Cancer Staging Manual provides new tools for recording and using stage in the medical record and the cancer registry, to support electronic capture of these data, and to foster the integration of other prognostic and response predictive factors with stage. These include a new "staging form" included in each disease chapter in the manual, an electronic version of the form, and a new tool for electronic capture of staging data and derivation of stage being released in late 2009.

The AJCC is also working closely with the cancer surveillance community and specialty organizations to define and implement clinical instruments that collect data in the format needed to transmit stage, store, and use stage and prognostic data. Beginning in 2004, the AJCC and its cancer surveillance partners implemented a data collection tool across all US hospital and population registries for cancer stage information, called the Collaborative Stage Data Collection System (CS). ${ }^{3}$ This uses a standardized data dictionary to collect information on T, N, M, and site-specific prognostic and predictive factors. The CS system includes the computer algorithms to derive TNM stage. The CS system is built into all cancer registry software systems in the USA. The primary data and derived stage are stored locally at the hospital registry and are also transmitted to central registries including state registries, SEER, and the National Cancer Data Base (NCDB). This data collection and storage tool may be expanded in the future to incorporate new factors relevant to cancer prognosis. It is also designed for an interoperable electronic environment to allow use of the CS primary staging elements and the CSderived TNM cancer stage in other electronic platforms.

In parallel with the publication of the 7th edition of the AJCC Cancer Staging Manual, the CS system is undergoing a major revision to be compatible with TNM, and to enhance its utility. In addition, the College of American
Pathologists (CAP) and a team led by the Centers for Disease Control and Prevention (CDC) are revising the CAP Cancer Templates for reporting pathology on cancer specimens to collect core elements on tumor size, extension, nodal involvement, and metastases in the format needed for recording in the CS system. The CS system is currently being implemented in Canada, and organizations in other nations have inquired about adoption of this tool. Finally, it is expected that the CS system will be incorporated in the NCI's Cancer Bioinformatics Grid (caBIG) as the accepted standard for recording data on the extent of disease and stage.

Looking to the future, the AJCC is also working with leaders in the epidemiology and statistical modeling scientific communities to foster the use of prognostic and predictive models in clinical oncology. With an increasing plethora of prognostic tools available on the Internet, the AJCC will provide the key leadership to help developers and users to coordinate use of existing models. More importantly, the AJCC will provide the leadership to coordinate and sponsor development of new prognostic and predictive models, a process already underway under the leadership of the current AJCC chair, Carolyn Compton. These efforts will be enhanced through the standardization of data collection with the CS system, coordination with $\mathrm{CAP}$, and the electronic AJCC staging tools.

A key feature of the 7th edition of TNM is coordination with the UICC. This is critical to maintain a single, worldwide system for recording and communicating cancer stage. This is particularly important, as cancer becomes one of the leading causes of death around the world. UICC representatives participated on all of the AJCC disease site taskforces. There were major international collaborations for data collection and analysis to revise TNM, which included the establishment of international data collection efforts in a number of diseases. Among these were a worldwide collaboration and database for lung cancer staging led by the International Association for the Study of Lung Cancer, a Worldwide Esophageal Cancer Collaboration, and the melanoma taskforce. Other major international collaborations led to the unification of gastric cancer staging across Asia, Europe, and North America, and coordination with the International Federation of Gynecology and Obstetric (FIGO) for staging of gynecologic malignancies. The AJCC manual and UICC manual were fully coordinated to be sure any issues were coordinated. Finally, the AJCC and UICC carefully reviewed the rules for TNM staging to ensure a uniform and clear approach to staging.

The editorials that will appear in subsequent issues of the Annals of Surgical Oncology will summarize specific information on the revisions made in key disease sites. These changes are clearly presented using an improved format for the AJCC Cancer Staging Manual. The manual 
includes 57 chapters from 16 taskforces that included 266 individuals from 5 continents and 11 countries. There are seven new chapters, as shown in Table 1 . In addition, there were major changes and expansion of the scope of a number of chapters, also shown in Table 1.

The AJCC taskforces were charged with making revisions to TNM based on the highest level of evidence possible. Where possible, taskforces used outcomes data from the NCDB and other existing and newly developed datasets. Where such data were more limited, they also based changes on expert consensus. The taskforces were also charged with defining key nonanatomic prognostic factors in two classifications: those necessary to define stage, and those critical to defining treatment. The use of nonanatomic factors was allowed in the definition of stage groups, now termed "anatomic stage/prognostic groups" to highlight the prognostic significance of the groupings. Examples of use of nonanatomic factors include incorporation of prostate-specific antigen (PSA) and Gleason's score in defining groupings in prostate cancer. A key feature in the use of these nonanatomic factors is that, in each case, the factors are used to modify the anatomic stage grouping, and not to define the $\mathrm{T}, \mathrm{N}$, and $\mathrm{M}$. In addition, the grouping schemas provide for assigning a stage group when the nonanatomic factors are not available. This is critical to assure worldwide applicability. These factors are also incorporated into the CS data collection system version 2 (CSv2).

A number of disease taskforces identified the response of the cancer to systemic or radiation therapy as key prognostic information. These included breast cancer, rectal cancer, esophageal cancer, head and neck cancers, and others. A number of these sites include response to

TABLE 1 New and revised chapters in the AJCC cancer staging manual, 7th edition

New chapters
Mucosal Melanoma of the Head and Neck
Appendix
Gastrointestinal Stromal Tumors (GIST)
Neuroendocrine Tumors
Merkel Cell Carcinoma
Adrenal Gland
Ocular Adnexal Lymphoma
Major revisions to chapter structure
Intrahepatic Bile Ducts (separated from Liver)
Extrahepatic Bile Ducts, split into
Perihilar Bile Ducts
Distal Bile Ducts
Carcinoma of the Skin
Cutaneous Squamous Cell Cancer
Other Cutaneous Cancer

treatment as a prognostic factor for collection. To address this need, the CS system will be further revised to include data fields for collection of information on the extent of $\mathrm{T}$ and $\mathrm{N}$ before and after neoadjuvant treatment. This will allow standardized recording of pretreatment stage and posttreatment stage (defined using the prefix " $y$ ") and allow derivation of the response to treatment. This enhancement of CS will be implemented in 2011.

Another enhancement in the 7 th edition of the AJCC Cancer Staging Manual is expansion of the first chapter on "Purposes and Principles of Cancer Staging" to more clearly delineate the rules of TNM staging. It is expected that this detailed exposition of the rules will reduce variation in interpretation of staging rules and result in higherquality data. In addition, in collaboration with the UICC, key rules have been clarified and revised. An important example is the rule related to the timeframe in which data may be obtained for clinical and pathologic staging in relation to date of diagnosis and initiation of treatment. Another critical change is the elimination of the classification of "MX" for unknown status of metastatic disease. Inappropriate use of "MX" in the past has led to many cases being "unstageable." Moving forward, any case without clinical or pathologic evidence of metastases is to be classified as clinically M0 (cM0). Assignment of cM0 does not require any imaging or other evaluation beyond history and physical examination. Users of cancer staging are urged to carefully read chapter 1 of the AJCC Cancer Staging Manual and to maintain this for reference.

The appearance of the manual itself has been enhanced to make it more user-friendly. The manual uses enhanced type fonts plus color-coding for $\mathrm{T}, \mathrm{N}$, and $\mathrm{M}$ elements. Each chapter starts with a clear table of changes from the prior edition and a summary called "staging-at-a-glance." In addition, paper and electronic versions of staging forms are enhanced to improve utility. The manual will be followed by companion print and electronic publications, including a smaller AJCC Cancer Staging Handbook, the 2nd edition of the AJCC Cancer Staging Atlas, and the electronic staging forms and staging software application.

The AJCC believes that this 7th edition of the AJCC Cancer Staging Manual will enhance the utility of the TNM staging to patients and clinicians. We hope that this series of editorials in the Annals of Surgical Oncology will further assist clinicians in understanding staging, and the changes made in TNM. However, publication of the 7th edition is just the next step. The 7th edition is also a springboard to the exciting future of personalized cancer care using data to more specifically define prognosis and the potential for benefit from specific therapies. The AJCC will lead the charge to bring scientific advances to broad clinical use to provide these advantages to all cancer patients worldwide. 
All such efforts require the input of legions of people who deserve enormous credit for their efforts. Members of each taskforce are listed in the manual. In addition, the AJCC owes a deep debt of gratitude to the staff at the AJCC, the registrars and scientists in surveillance organizations working on CSv2, and the many around the world who have contributed. Finally, the AJCC expresses a special thanks to the former chair of the AJCC, Irvin Fleming, to whom the manual is dedicated. His vision over the last three decades led to many of the strategic decisions that led the AJCC to its current position, from where it is poised for an exciting future.

\section{REFERENCES}

1. Edge SB, Byrd DR, Compton CC, Fritz AG, Greene FL, Trotti A, editors. AJCC cancer staging manual (7th ed). New York, NY: Springer; 2010.

2. www.adjuvantonline.com. Accessed 26 Dec 2009.

3. www.cancerstaging.org. Accessed 26 Dec 2009. 